Claremont Colleges

Scholarship@ Claremont

All HMC Faculty Publications and Research

HMC Faculty Scholarship

6-26-2006

\title{
Hole Dynamics in Polymer Langmuir Films
}

James C. Alexander

Case Western Reserve University

Andrew J. Bernoff

Harvey Mudd College

Elizabeth K. Mann

Kent State University - Kent Campus

J. Adin Mann Jr.

Case Western Reserve University

LuZou

Kent State University - Kent Campus

\section{Recommended Citation}

Hole dynamics in polymer Langmuir films. James C. Alexander, Andrew J. Bernoff, Elizabeth K. Mann, J. Adin Mann, Jr., and Lu Zou, Phys. Fluids 18, 062103 (2006).

This Article is brought to you for free and open access by the HMC Faculty Scholarship at Scholarship @ Claremont. It has been accepted for inclusion in All HMC Faculty Publications and Research by an authorized administrator of Scholarship @ Claremont. For more information, please contact scholarship@cuc.claremont.edu. 


\title{
Hole dynamics in polymer Langmuir films
}

\author{
James C. Alexander ${ }^{a}$ \\ Department of Mathematics, Case Western Reserve University, Cleveland, Ohio 44106 \\ Andrew J. Bernoff ${ }^{\text {b) }}$ \\ Department of Mathematics, Harvey Mudd College, Claremont, California 91711 \\ Elizabeth K. Mann ${ }^{\mathrm{c})}$ \\ Department of Physics, Kent State University, Kent, Ohio 44242 \\ J. Adin Mann, Jr. ${ }^{\text {) }}$ \\ Department of Chemical Engineering, Case Western Reserve University, Cleveland, Ohio 44106 \\ Lu Zou ${ }^{\mathrm{e}}$ \\ Department of Physics, Kent State University, Kent, Ohio 44242
}

(Received 3 August 2005; accepted 12 May 2006; published online 28 June 2006)

\begin{abstract}
This article develops a model for the closing of a gaseous hole in a liquid domain within a two-dimensional fluid layer coupled to a Stokesian subfluid substrate, and compares this model to experiments following hole dynamics in a polymer Langmuir monolayer. Closure of such a hole in a fluid layer is driven by the line tension at the hole boundary and the difference in surface pressure within the hole and far outside it. The observed rate of hole closing is close to that predicted by our model using estimates of the line tension obtained by other means, assuming that the surface pressure in the gas is negligible. This result both supports the model and suggests an independent means of determining the line tension. Unlike most previous hydrodynamics models of Langmuir films, the closing of a hole necessarily involves vertical motion of the underlying incompressible fluid. Fluid is dragged along with the liquid monolayer towards the center of the hole, and must plunge away from the surface. An explicit expression is found for this vertical fluid flow in the bulk substrate. () 2006 American Institute of Physics. [DOI: 10.1063/1.2212887]
\end{abstract}

\section{INTRODUCTION}

A Langmuir film is a molecularly thin layer at the airwater interface. The molecules are trapped at the interface by a balance of interactions between the monolayer molecules with each other and with the substrate. Depending on the surface density, the film may form quasi-two-dimensional analogs of gases, liquids, liquid crystals, or solids. The system is quasi-two-dimensional in the sense that the motion of molecules is confined essentially to the plane of the interface (often called the Gibbs plane). A thermodynamic equation of state for the monolayer relates the surface pressure $\Pi$ and the surface density $\bar{\rho}$. As in the three-dimensional case, the film will separate at intermediate average densities, into gas and liquid phases for example, forming a distribution of separate domains at the surface.

The phase coexistence region has drawn considerable experimental attention. A wide variety of morphologies and dynamical behavior is observed. ${ }^{1}$ The domain size is potentially controllable over a wide range of sizes from the nano to the micro scales, so that arrays of domains with different physical and chemical properties can be formed by transferring the Langmuir layer to a solid substrate. There is growing

\footnotetext{
${ }^{a)}$ Electronic mail: james.alexander@case.edu

b)Electronic mail: ajb@math.hmc.edu

${ }^{c)}$ Electronic mail: emann@kent.edu

d)Electronic mail: j.mann@case.edu

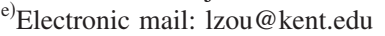

recognition of the functional importance of domains in biological cell membranes ${ }^{2-6}$ where domains may sequester proteins needed for signaling or provide structural conditions for shape changes. Langmuir films provide a controlled model for such domain formation.

The size distribution of domains within the Langmuir film often depends on film history, and equilibration is slow. Thus, a simple hydrodynamic model for changing domain size was developed. ${ }^{7}$ However, recent experiments ${ }^{8}$ on hole closing in fluid monolayers disagree with this model, and those authors suggested that further theoretical treatment is necessary.

Hole closing within polymer Langmuir films was first noted more than 10 years ago. ${ }^{9,10}$ The polymer, poly(dimethylsiloxane) or PDMS, has served as a model polymer for many experimental studies on wetting for example. It is unusually flexible, with a particularly low surface viscosity. On the water surface, it forms a monolayer one monomer $(\sim 0.7 \mathrm{~nm})$ thick, $^{9,11,12}$ so that the number of molecules in a full monolayer is inversely proportional to the number of monomer units in an average polymer chain. With less than full coverage by this monolayer, the polymer phase separates into a dense two-dimensional (2D) liquid phase and a very dilute 2D gas (see Fig. 1). The surface pressure at gas-liquid coexistence is unmeasurably low. The liquid phase has a very high elasticity, and can thus be considered incompressible. The case for the gaseous phase is less clear, as discussed in detail in Sec. II A, but it is likely to be closer to the opposite 


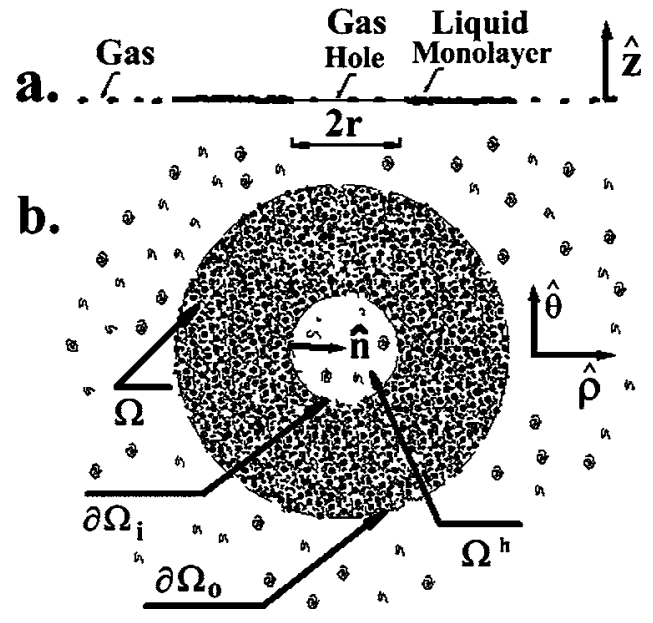

FIG. 1. (a) A side-on view of a gas-phase polymer hole in a polymer liquid domain. The whole monolayer is on a water substrate. (b) A view from the top of the gas-phase hole $\left(\Omega^{h}\right)$ and the liquid phase domain $\Omega$. The inner boundary is $\partial \Omega_{i}$ and the normal $\hat{n}$ is defined to be outwards from the denser, liquid phase on $\partial \Omega_{i}$. The outer boundary of the domain is $\partial \Omega_{o}$. The origin of the coordinate system is the center of $\Omega^{h}$; the radial distance to $\partial \Omega_{i}$ is $r$ and to $\partial \Omega_{o}$ is $R$.

limit of zero elasticity, especially for a polymer with a large molecular weight, which leads to a very low number density on the surface. This consideration is a major motivation for the current model.

At ambient humidity, the boundary between very large scale $(>\mathrm{cm})$ domains of polymer liquid and polymer gas showed stable holes $(5-100 \mu \mathrm{m})$ in the liquid phase, often with a $2 \mathrm{D}$ foam-like morphology. When a cover was placed on a monolayer showing holes, so that the humidity of the layer environment increased towards saturation, the area of the holes decreases more or less exponentially. ${ }^{10}$ The importance of humidity to this phenomenon was demonstrated by blowing nitrogen gas across a covered monolayer. Holes opened up in the presence of dry nitrogen and closed with water-saturated nitrogen. This closure, possibly due to temperature gradients because of evaporative effects, took place far from equilibrium, as the humidity above the layer increased continuously. Here, in order to avoid this problem, we artificially open holes in the monolayer, and watch their collapse.

More recently, Khattari et al. ${ }^{8}$ examine hole closing in both fluid and liquid crystalline phases within several types of monolayers. They placed themselves above the phase coexistence pressure, in a single-phase regime, and burned holes in the monolayer with an IR laser. They assumed that the temperature equilibrated much more quickly than other processes, and that the pressure in the hole was the same as the pressure of the gas in coexistence with the condensed phase, thus much lower than that of the surrounding condensed phase. The surface pressure difference would then drive the hole closing, and the line tension would be negligible. This model worked qualitatively when the condensed monolayer acted as a solid. However, the rate of hole closing within a liquid monolayer was more than six orders of magnitude lower than this simple model would suggest. At these rates, the line tension should no longer be neglected. The authors suggest that their liquid phase might not be sufficiently incompressible for their model to apply. Significant compressibility is unlikely for any liquid monolayer for the slow speeds they observe, and indeed, the elasticity of their liquid phase is measured to be $\sim 20 \mathrm{mN} / \mathrm{m},{ }^{13}$ more than four orders of magnitude greater than required for incompressibility (see Sec. II A). However, compressibility could be important in the gaseous phase.

Our goal is thus to develop a model for hole closing in a different limit, assuming that the gaseous center is dilute, but including both pressure and line tension. Furthermore, we model a monolayer at liquid-gas coexistence. This model for the closing of a gaseous hole in a liquid domain within a two-dimensional liquid layer coupled to a bulk fluid substrate is compared to experiments following such holes in a polymer monolayer. The hydrodynamics of domain equilibration requires consideration of the surface fluids, the fluid substrate, and the coupling between them. The net attraction between monolayer molecules, in the environment of the interface, leads to a line tension, or energy per unit length $\lambda$, associated with the boundary between domains. Lateral intermolecular forces include short-range van der Waals forces, but also long-range dipolar repulsion due to the alignment of the effective molecular dipole moments by the interface. Above a critical size $\mathrm{s}^{12,14}$

$$
R_{c}=\frac{\delta}{8} \exp \left(\frac{2 \pi \lambda}{\varepsilon_{0}(\Delta V)^{2}}+\frac{10}{3}\right)
$$

where $\delta$ is a characteristic molecular length, $\lambda$ is the line tension, $\varepsilon_{0}$ is the vacuum dielectric constant, and $\Delta V$ is the contrast in surface potential between the two phases. The long-range electrostatic forces distort domains from the circular shape. Using literature values of the surface potential and line tension $\Delta V=0.2 \mathrm{~V}$ (Ref. 15) and $\lambda=1.1 \mathrm{pN}$ (Ref. 16), and estimating the molecular length as the monomer size $\sim 0.4 \mathrm{~nm}$, we find $R_{c}=0.5 \mathrm{~m}$, which is seven times larger than the width of the trough. At dimensions much smaller than $R_{c}$, the major effect of the long range forces is to renormalize the line tension as ${ }^{14}$

$$
\lambda_{\mathrm{eff}}=\lambda-\mu^{2}\left(\ln \frac{L_{*}}{L_{m}}+I_{s}\right)
$$

where $\mu$ is the effective dipole moment density difference between phases, given in terms of the measured surface potential contrast as ${ }^{12} \mu^{2}=\varepsilon_{0}(\Delta V)^{2} / 2 \pi, L_{*}$ is a typical domain length scale, $L_{m}$ is some molecular scale (often taken to be the thickness of the layer or the average distance between molecules in the layer), and $I_{s}$ is a term depending only on shape (generally negligible until the effective line tension approaches zero). Since the main correction to the line tension is logarithmic, the line tension can often be taken as a constant over a large range of length scales. In that approximation, the line tension leads directly to closure of the hole, in order to minimize the line energy. Closure of such a hole in a liquid layer is thus driven by the line tension as well as the difference in pressure within the hole and outside it. Note that this process is one of the mechanisms for Ostwald rip- 


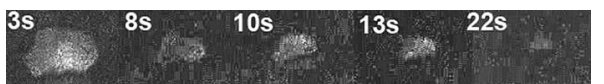

FIG. 2. The evolution with time of a hole (brighter area), or 2D polymer gas, within a polymer liquid (darker area) monolayer. The images are taken by Brewster angle microscopy (BAM). Variations in the beam intensity are off the same order of magnitude as the contrast for these two very thin films. Contrast has been improved with Photoshop ${ }^{\mathrm{TM}}$. Each image is $0.27 \mathrm{~mm}$ $\times 0.44 \mathrm{~mm}$

ening, but that grain coarsening would also require domain coalescence, which is not observed. ${ }^{17}$

Several authors ${ }^{18,19}$ have considered the case where the surface fluids are incompressible and with the same surface viscosity. In this case, solutions of the hydrodynamic equations with strictly horizontal flow exist. This incompressibility condition does not necessarily hold for a gaseous layer. A contrast in surface viscosity or compressibility leads to flow perpendicular to the surface. Hole closing always leads to such flow, regardless of viscosity contrast, since as the hole closes, the underlying fluid is dragged with the monolayer towards the center of the hole. That fluid has to go somewhere. The only place for it to go is down into the subfluid. Hence there is a vertical component to the flow.

The model we develop here assumes that the surface viscosity is negligible in both domains, which is often realistic and has been demonstrated explicitly ${ }^{16}$ over the size range of interest for the polymer layers considered here. The liquid phase is considered to be incompressible, while, unlike previous models, the compressibility of the gaseous phase is considered to be null. In the current model, longrange forces are ignored. This initial model also ignores accretion of molecules from the hole $\Omega^{h}$ onto the liquid phase boundary $\partial \Omega_{i}$, or inversely, evaporation from the liquid phase boundary into the hole, because we expect the concentrations of the polymer to be very low in the gas phase. We do not include nucleation within the hole since nucleation of liquid domains within the hole is not observed. We find an explicit relation between the rate of area decrease, the line tension and bulk viscosity, and the pressure difference between the domain interior and the reference pressure far from the domains. We also find explicit expressions for flow in the bulk fluid. An appendix ${ }^{20}$ considers the density within the hole as a function of time, which can be used, if necessary, to consider the accretion or evaporation of the polymer from the boundary. In our experiments, the polymer density in the hole did not visibly change during the hole closing.

The rate of hole closing given by this model is compared with the rate observed in polymer monolayers, see Fig. 2. From this rate we deduce a line tension, which is close to that determined by the characteristic relaxation time of deformed domains. ${ }^{16}$ Determining the line tension has been difficult, and relatively few examples exist in the literature. The existence of a line tension means that small isolated domains are round in equilibrium, and also that deformed domains relax to this shape. This relaxation has served for estimates of the line tension in a few systems, including phospholipid/cholesterol mixtures, ${ }^{21}$ polymer mono and multilayers, ${ }^{12,16}$ smectic liquid crystalline layers, ${ }^{22}$ and fatty acids. ${ }^{23,24}$ Other means of determining the line tension would be welcome. Furthermore, many of the assumptions of the models used to analyze the data may be crude; that both surface fluids are incompressible, for example. The agreement between the line tension calculated from our model and that determined by the relaxation experiments result both supports the model, including the assumption of negligible elasticity for the gas phase, and suggests an independent means of determining the line tension.

\section{FORMULATION}

\section{A. Basic approximations}

As the subfluid in the experiment is usually water or some aqueous solution, we assume its evolution will be governed by the Navier-Stokes equation for an incompressible fluid characterized by its density $\rho^{\prime}$ and viscosity $\eta^{\prime}$ (which under the experimental conditions reduces to the Stokes equation, see below). Moreover, following previous authors, we will also assume that the polymer monolayer can be modelled as a two-dimensional inviscid fluid with associated surface density $\bar{\rho}$. Direct measurements of the relaxation time as a function of bulk viscosity ${ }^{16}$ for this polymer (PDMS) on water demonstrated that relaxation was dominated by viscosity in the subfluid for domains in the 5-100 $\mu \mathrm{m}$ range (surface viscosity should play a role in sufficiently small domains, but such domains would be below experimental resolution). This is likely to be true for many $2 \mathrm{D}$ fluid phases. The monolayer and the subfluid are coupled together at the surface by the applied stress balance-the subfluid's surface tension acts in the normal direction, the line tension acts in the tangent to the surface (and normal to the domain's boundary), and viscous normal and tangential stresses can be found in both directions.

We further assume that the polymer liquid is incompressible but that the polymer gas cannot support stress (Gibbs elasticity $K_{g} \equiv \bar{\rho} \partial \pi / \partial \bar{\rho}=0$ ). A surface phase may be considered incompressible if the elasticity $K_{g} \gg \lambda L$ $=10^{-12} \mathrm{~N} / L$, where $L$ is the characteristic size of the domain. Generally the accessible range of domain size is $2 \mu \mathrm{m}<L$ $<5 \mathrm{~mm}$, depending on the experimental setup. For the liquid phase, the elasticity has been directly measured: $K_{g}=40 \mathrm{mN} / \mathrm{m} .{ }^{9}$ The liquid phase can certainly be considered incompressible. The case of the polymer gas is uncertain, since experimental data give only an upper limit for its elasticity, still well above the $K_{g} \sim \lambda / L$ transition between the two regimes. For PDMS, direct measurements of the elasticity of the gas phase cannot distinguish it from nothing at all, ${ }^{9}$ but this limit is only $\sim \mathrm{mN} / \mathrm{m}$, so we turn to surface pressure measurements for a more accurate limit. The surface pressure at gas-liquid coexistence is itself immeasurably low $(\Pi<0.02 \mathrm{mN} / \mathrm{m})$. This is expected, because of the large molecular weight of the polymer (here 400 monomer units); even the surface pressure from the ideal gas law would be very low $\left(\Pi<5 \times 10^{-3} \mathrm{mN} / \mathrm{m}\right.$, from the experimental limit for the polymer concentration in the $2 \mathrm{D}$ gas, less than a tenth of that in the 2D liquid; ${ }^{12}$ the actual concentration may be much less). Data from lower molecular weights, ${ }^{25}$ where surface pressure is measurable, extrapolate 
to a much smaller surface pressure: $\Pi \ll 5 \times 10^{-4} \mathrm{mN} / \mathrm{m}$. The elasticity $K_{g}$ of the gas phase is less than the surface pressure, or $K_{g} \ll 5 \times 10^{-4} \mathrm{mN} / \mathrm{m}$. Our upper limit for the compressibility of the gaseous phase is thus too high to conclude, but plausibly, the phase cannot be considered incompressible. Thus, we will consider the opposite limit of zero elasticity for holes in the liquid. This limit is most likely to hold in a gaseous polymer monolayer, because of its low number density.

We propose the ansatz that the collapse of a hole is driven by the line tension $\lambda$, of the polymer film and that the energy is dissipated by the viscosity, $\eta^{\prime}$, of the subfluid. Experimentally, the dynamics of a hole are determined by estimating the area as a function of time from a pixelated photographic image. To scale and nondimensionalize the model, we use the area $A_{*}$ of a pixel $\left(\approx 13 \mu \mathrm{m}^{2}\right)$ as a fiducial area. From $A_{*}$, we set characteristic length, time and mass,

$$
\begin{aligned}
& L_{*}=\sqrt{A_{*}}, \\
& T_{*}=\frac{\eta^{\prime}\left(L_{*}\right)^{2}}{\lambda}, \\
& M_{*}=\eta^{\prime} L_{*} T_{*}=\frac{\left(\eta^{\prime}\right)^{2}\left(L_{*}\right)^{3}}{\lambda},
\end{aligned}
$$

respectively. The camera limits the accessible domain size to $5<L / L_{*}<500$.

\section{Stokesian subfluid}

We set axisymmetric (cylindrical) coordinates $\rho=\sqrt{x^{2}+y^{2}}, \theta, z$, with corresponding unit vectors $\hat{\rho}, \hat{\theta}, \hat{z}$. We consider holes that are small with respect to the surrounding polymer film, and also with respect to the depth of the fluid. Accordingly we posit a Stokesian subfluid in the region $\{z \leq 0,0 \leq \rho<\infty\}$. This is a first approximation to what we observe (see Fig. 2), capturing the basic physics. We assume the hole is circular, centered at $(\rho, z)=(0,0)$, and that the evolution is axisymmetric. Thus let

$$
\mathbf{u}(\rho, z, t)=u(\rho, z, t) \hat{\rho}+w(\rho, z, t) \hat{z}
$$

be the nondimensional velocity and $P(\rho, z, t)$ be the nondimensional pressure. In the bulk of the subfluid, we assume the nondimensional fluid velocity is incompressible,

$$
\nabla \cdot \mathbf{u}=0
$$

and satisfies the nondimensional Navier-Stokes equations

$$
\operatorname{Re}\left(\mathbf{u}_{t}+\mathbf{u} \cdot \nabla \mathbf{u}\right)=-\nabla P+\nabla^{2} \mathbf{u},
$$

where the Reynolds number,

$$
\operatorname{Re}=\frac{\rho^{\prime} \lambda}{\left(\eta^{\prime}\right)^{2}}
$$

is assumed to be small; for a water substrate and a monolayer, with $\lambda \lesssim 10^{-9}$ Newtons we find that $\operatorname{Re} \leq 10^{-2}$. Consequently, the subfluid velocity satisfies the Stokes equations

$$
\nabla P=\nabla^{2} \mathbf{u}
$$

at leading order. The fluid is incompressible, and thus there is a stream function $\psi(\rho, z, t)$ with

$$
\mathbf{u}=\nabla \times(\psi \hat{\theta})=-\psi_{z} \hat{\rho}+\frac{1}{\rho} \partial_{\rho}(\rho \psi) \hat{z} .
$$

Taking the curl of (10) and using (11) (and the fact that vector Laplacian and curl commute),

$$
\begin{aligned}
0 & =\nabla \times \nabla P=\nabla^{2}(\nabla \times \mathbf{u}) \\
& =\nabla^{2}(\nabla \times(\nabla \times(\psi \hat{\theta}))) \\
& =-\nabla^{2}\left(\nabla^{2}(\psi \hat{\theta})\right) ;
\end{aligned}
$$

the last from the general identity $\nabla \times(\nabla \times \mathbf{w})=\nabla(\nabla \cdot \mathbf{w})$ $-\nabla^{2} \mathbf{w}$. Here $\nabla^{2}$ is the vector Laplacian $\nabla^{2}(\psi \boldsymbol{\theta})=\left(\mathcal{L}_{\rho} \psi\right.$ $\left.+\psi_{z z}\right) \hat{\theta}$, where

$$
\mathcal{L}_{\rho}=\partial_{\rho}^{2}+\frac{1}{\rho} \partial_{\rho}-\frac{1}{\rho^{2}} .
$$

Thus Stokes equation (10) reads

$$
\left(\mathcal{L}_{\rho}+\partial_{z}^{2}\right)^{2} \psi=0 .
$$

\section{Surface conditions}

The Langmuir film on the surface is coupled to the subfluid. As the surface tension (which tends to flatten the surface) is much stronger that the viscous stresses at the surface we assume that the surface is flat to leading order. Thus the vertical velocity at the surface vanishes. That is [with reference to (6), here and in (15)],

$$
w(\rho, 0, t)=\left.\frac{1}{\rho} \partial_{\rho}(\rho \psi)\right|_{z=0}=0 .
$$

In addition, continuity of the tangential velocity at the surface is used to compute the surface fluid velocity. That is, if $\mathbf{U}(\rho, t)=U(\rho, t) \hat{\rho}$ is the surface velocity, by (11),

$$
U(\rho, t)=u(\rho, 0, t)=-\psi_{z}(\rho, 0, t) .
$$

Suppose the fluid Langmuir film is in the region $\Omega=\{(\rho, \theta): r \leq \rho \leq R\}$. The hole is the region $\Omega^{h}=\{(\rho, \theta): 0$ $\leq \rho<r\}$ and the complement $\bar{\Omega}$ of $\Omega$ is the region $\{(\rho, \theta): 0 \leq \rho<r\} \cup\{(\rho, \theta): R<\rho\}$ (see Fig. 1). We are interested in the situation $R \gg r$; i.e., asymptotically $R=\infty$. However, it is useful to be able to explicitly reference the pressure in the far-field domain $\{(\rho, \theta): \rho>R\}$. Moreover, if the polymer domain is finite in extent, the force it applies at the surface is localized and the response of the subfluid decays algebraically in $r$ and exponentially in $z$; consequently, we can assume as that the velocity $\mathbf{u}$ and its gradients decay uniformly to 0 at $\infty$ :

$$
|\mathbf{u}|, \quad|\nabla \mathbf{u}|, \quad\left|\nabla^{2} \mathbf{u}\right| \rightarrow 0 \quad \text { as } \rho^{2}+z^{2} \rightarrow \infty .
$$

We also can assume the stream function $\psi$ is bounded and that

$$
|\nabla \psi|, \quad\left|\nabla^{2} \psi\right|, \quad\left|\nabla\left(\nabla^{2} \psi\right)\right| \rightarrow 0 \quad \text { as } \rho^{2}+z^{2} \rightarrow \infty .
$$


Let $\Pi(\rho, t)$ denote the pressure in the Langmuir layer. The tangential surface stress $\mathbf{F}_{s}=-U_{z} \hat{\boldsymbol{\rho}}=\psi_{z z} \hat{\boldsymbol{\rho}}$. Note that $\nabla \Pi=\Pi_{\rho}$. Thus the assumption of a hydrostatic Langmuir layer and stress-free $\bar{\Omega}$ implies

$$
\psi_{z z}(\rho, 0, t)= \begin{cases}\Pi_{\rho} & \text { in } \Omega, \\ 0 & \text { in } \bar{\Omega} .\end{cases}
$$

Denote the pressure in the hole $\Omega^{h}$ by $\Pi_{i}$ (for inner) and the pressure in the region $\{(\rho, \theta): R<\rho\}$ by $\Pi_{o}$ (for outer). At a point on the boundary between the planar liquid and gaseous phases, the difference in pressures is $\lambda / \kappa$, where $\kappa$ is the (signed) curvature of the boundary at the point. The nondimensionalized line tension is 1 . Then we obtain the line tensions on the inner and outer boundary of $\Omega$ as

$$
\begin{aligned}
& \Pi(r, t)-\Pi_{i}=-\frac{1}{r}, \\
& \Pi(R, t)-\Pi_{o}=-\frac{1}{R} .
\end{aligned}
$$

Let $\nabla_{\perp} \cdot$ denote the (2D) surface divergence. Incompressibility in the fluid Langmuir layer $\Omega$ implies

$$
\nabla_{\perp} \cdot U(\rho, t)=-\left.\frac{1}{\rho} \partial_{\rho}\left(\rho \psi_{z}\right)\right|_{z=0}=0 .
$$

This equation, together with (15), implies that

$$
U(\rho, t)=-\psi_{z}(\rho, 0, t)=\frac{-q(t)}{\rho},
$$

for some function $q(t)$. Let $A=A(t)$ be the area of the hole $\Omega^{h}$. Then

$$
A_{t}=2 \pi r r_{t}=2 \pi r U(r, t)=-2 \pi q(t) .
$$

\section{B. Derivations}

The formulation in the previous section leads to a focus on the stream function $\psi(\rho, z, t)$ and related functions such as $q(t)$. The purpose of this section is to develop explicit formulas for these functions, beginning with Stokes equation (13), and leading to a complete description of the flow. Perhaps the result most relevant to experimental predictions is the formula (38) below, derived from (23), for the behavior of the area of the hole. The determination of $\psi(\rho, z, t)$, see (41) and (43) below, leads to a complete description of the flow in the subfluid, see Figs. 3 and 4.

We turn to the derivation. Consider the (first-order) Hankel transform

$$
F(\ell, z, t)=\int_{0}^{\infty} \rho \psi(\rho, z, t) J_{1}(\ell \rho) d \rho,
$$

where $J_{i}$ is the $i$ th order Bessel function of the first kind. Let $f(\ell, z, t)=\ell F(\ell, z, t)$, so that

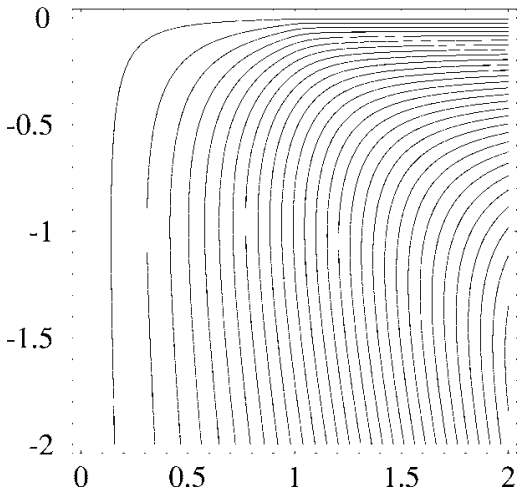

FIG. 3. A vertical cross section of flow lines in the subfluid, for $\Pi=0$. The axes are $0 \leq \rho / r \leq 2$ and $0 \geq z / r \geq-2$. The angular coordinate is suppressed. Thus the top of the graph is the surface of the subfluid and the hole boundary is at radius $\rho / r=1$. The flow is down and to the left. The fluid moves more rapidly when the flow lines are closer together. The most rapid movement is at the boundary of the hole. The flow at $\rho=0$ (the left of the graph) is vertical (by symmetry) downward, and maximal when $z / r=-1$.

$$
\psi(\rho, z, t)=\int_{0}^{\infty} f(\ell, z, t) J_{1}(\ell \rho) d \ell .
$$

We first show that $f(\ell, z, t)=B(\ell, t) z e^{\ell z}$ for some function $B(\ell, t)$. To this end, substitute (24) into (13) and note that $\mathcal{L}_{\rho} J_{1}(\ell \rho)=-\ell^{2} J_{1}(\ell \rho)$. Hence $f(\ell, z, t)$ satisfies the equation $\left(\partial_{z}^{2}-\ell^{2}\right)^{2} f=0$, which has solutions

$$
\begin{aligned}
f(\ell, z, t)= & A(\ell, t) e^{\ell z}+B(\ell, t) z e^{\ell z}+C(\ell, t) e^{-\ell z} \\
& +D(\ell, t) z e^{-\ell z} .
\end{aligned}
$$

The conditions (17) imply $C, D=0$. Equation (14) implies $\psi(\rho, 0, t)=E(t) / \rho$, which is unbounded unless $\psi(\rho, 0, t)=0$, which in turn implies $A=0$. This establishes the form of $f$ and we obtain the result

$$
\psi(\rho, z, t)=\int_{0}^{\infty} B(\ell, t) z e^{\ell z} J_{1}(\ell \rho) d \ell .
$$

We next determine $B$. With reference to $(22)$, let $B(\ell, t)$ $=q(t) g(\ell, t)$. We claim

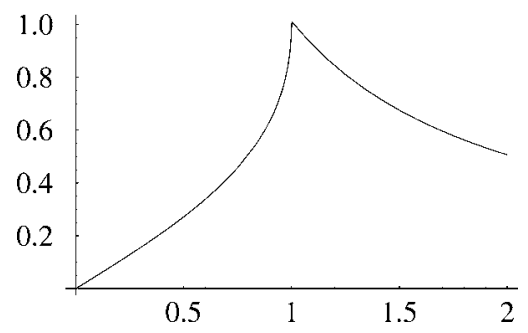

FIG. 4. The nondimensional fluid speed at the surface as a function of the radial coordinate $\rho / r$, for the case $\Pi=0$. The horizontal range is $0<\rho / r$ $<2$. The hole is in the region $0<\rho / r<1$ and the region to the right is the Langmuir film $\Omega$. The velocity is radially inwards. 


$$
\begin{aligned}
& \int_{0}^{\infty} \ell g(\ell, t) J_{1}(\ell \rho) d \ell=0 \quad \text { in } \bar{\Omega}, \\
& \int_{0}^{\infty} g(\ell, t) J_{1}(\ell \rho) d \ell=\frac{1}{\rho} \quad \text { in } \Omega .
\end{aligned}
$$

To establish (27), differentiate (26) twice with respect to $z$ and use the second equation of (18). To establish (28), differentiate (26) once with respect to $z$ and use (22). Rescale these equations by $r$. That is, let $\rho=r \zeta, \ell=k / r$. Note that (27), (28) are independent of $t$, except via the $t$ dependence of $\Omega$. Thus, let $\zeta^{*}=R / r$. Write $g(\ell, t)=G\left(k, \zeta^{*}\right)$. Then $G$ satisfies the triple integral equations

$$
\begin{aligned}
& \int_{0}^{\infty} k G\left(k, \zeta^{*}\right) J_{1}(k \zeta) d k=0, \quad 0<\zeta<1, \\
& \int_{0}^{\infty} G\left(k, \zeta^{*}\right) J_{1}(k \zeta) d k=\frac{1}{\zeta}, \quad 1<\zeta<\zeta^{*}, \\
& \int_{0}^{\infty} k G\left(k, \zeta^{*}\right) J_{1}(k \zeta) d k=0, \quad \zeta^{*}<\zeta<\infty .
\end{aligned}
$$

These equations determine $G$, and in fact, we find an exact solution in the limit $\zeta^{*} \rightarrow \infty$. We find

$$
\begin{aligned}
\int_{r}^{R} \Pi_{\rho} d \rho & =\int_{r}^{R} \psi_{z z} d \rho \quad[\text { from }(18)] \\
& =2 \int_{0}^{\infty} \ell B(\ell, t) \int_{r}^{R} J_{1}(\ell \rho) d \rho d \ell \quad \text { [differentiating (26)] } \\
& =-\left.2 \int_{0}^{\infty} B(\ell, t) J_{0}(\ell \rho) d \ell\right|_{\rho=r} ^{\rho=R}\left(J_{0}^{\prime}=-J_{1}\right) \\
& =-\left.2 q(t) \int_{0}^{\infty} g(\ell, t) J_{0}(\ell \rho) d \ell\right|_{\rho=r} ^{\rho=R}[\text { from (22)] } \\
& =-\left.\frac{2 q(t)}{r} \int_{0}^{\infty} G(k, \zeta) J_{0}(k \zeta) d k\right|_{\zeta=1} ^{\zeta=\zeta^{*}} .
\end{aligned}
$$$$
\int_{0}^{\infty} G\left(k, \zeta^{*}\right) J_{1}(k \zeta) d k=\int_{0}^{\infty} \frac{\sin k}{k} J_{1}(k \zeta) d k=\frac{1}{\zeta}, \quad 1<\zeta<\infty .
$$

For reference below, ${ }^{20}$ we note ${ }^{26}$

$$
\begin{aligned}
\int_{0}^{\infty} G\left(k, \zeta^{*}\right) J_{0}(k) d k & \rightarrow \int_{0}^{\infty} G(k, \infty) J_{0}(k) d k \\
& = \begin{cases}\pi / 2, & 0<p \leq 1, \\
\csc ^{-1} p, & p>1 .\end{cases}
\end{aligned}
$$

To determine $q(t)$, we compute

Thus the asymptotic computations below [such as (35)] obasymptotic results suffice completely for experimental correspondence). The technical computation is sketched in Appendix $\mathrm{A}^{20}$ The asymptotic case, $\zeta^{*}=R / r=\infty$ can be verified directly: $:^{26}$

$\int_{0}^{\infty} k G\left(k, \zeta^{*}\right) J_{1}(k \zeta) d k=\int_{0}^{\infty} \sin k J_{1}(k \zeta) d k=0, \quad 0<\zeta<1$, 


$$
q(t)=\frac{1}{\pi}(1-r \Delta \Pi)
$$

and consequently, from (23),

$$
A_{t}=-2(1-r \Delta \Pi)=-2\left(1-\sqrt{\frac{A}{\pi}} \Delta \Pi\right) .
$$

We can also calculate a closed form for $\psi(\rho, z, t)$, which in turn yields the flow in the fluid. We are restricted to $\zeta^{*}=\infty$. Thus

$$
B(\ell, t)=q(t) g(\ell, t)=q(t) \frac{\sin \ell r}{\ell r},
$$

so that from (26)

$$
\psi(\rho, z, t)=q(t) \int_{0}^{\infty} \frac{\sin \ell r}{\ell r} z e^{\ell z} J_{1}(\ell \rho) d \ell .
$$

The integral in (40) can be evaluated in closed form. If $s$, $0<s<1$, is the (unique) positive solution of

$$
r^{2}=\frac{\rho^{2}}{1-s^{2}}-\frac{z^{2}}{s^{2}}
$$

( $s$ is constant along rectangular hyperbolas), namely

$$
s=\frac{1}{r \sqrt{2}} \sqrt{r^{2}-\rho^{2}-z^{2}+\sqrt{4 r^{2} z^{2}+\left(r^{2}-\rho^{2}-z^{2}\right)^{2}},}
$$

then $^{26}$

$$
\int_{0}^{\infty} \frac{\sin \ell r}{\ell r} z e^{\ell z} J_{1}(\ell \rho) d \ell=\frac{z}{\rho}(1-s) .
$$

Thus from (40)-(42), the stream function

$$
\psi(\rho, z, t)=\frac{z}{\rho} q(t)(1-s)
$$

and thus the flow can be determined (as contour lines of $\rho \psi$ ), see Fig. 3. From (11) the velocity field can be determined as

$$
\begin{aligned}
U(\rho, t) & =-\psi_{z}(\rho, 0, t) \\
& =-q(t) \times \begin{cases}\frac{1}{\rho}\left[1-\sqrt{1-\left(\frac{\rho}{r}\right)^{2}}\right], & \rho<r, \\
\frac{1}{\rho}, & \rho>r,\end{cases}
\end{aligned}
$$

see Fig. 4.

We can also compute the pressure field in the subfluid; using (10) and (40), and we verify

$$
P(\rho, z, t)=q(t) \int_{0}^{\infty} 2 e^{\ell z} \frac{\sin \ell r}{r} J_{0}(\ell \rho) d \ell,
$$

see Fig. 5.

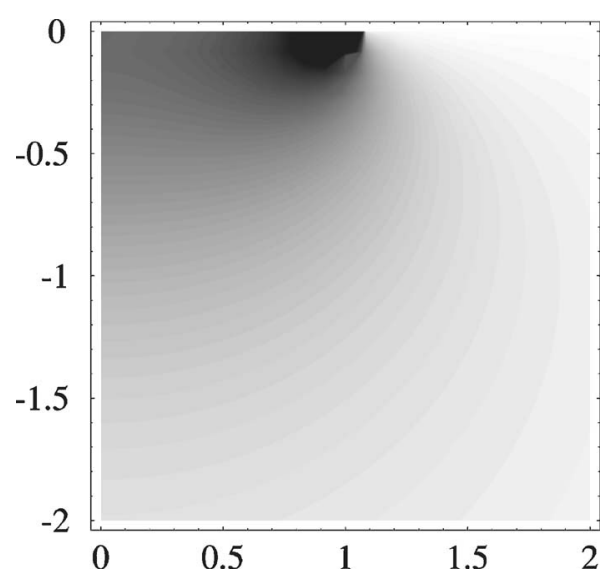

FIG. 5. A vertical cross section of the nondimensional pressure field for the case of $\Pi=0$. The horizontal axis is $0<\rho / r<2$ and the vertical axis is -2 $<z / r<0$. Darker shading indicates higher pressure.

\section{EXPERIMENTS}

\section{A. Experimental methods}

Poly (dimethylsiloxane) $\quad\left(\mathrm{PDMS}=\left(\mathrm{OSi}\left(\mathrm{CH}_{3}\right)^{2}\right)_{N}, \quad M_{w}\right.$ $=31600$ and polydispersity $\left.M_{w} / M_{N}=1.06 ; N=427\right)$ was purchased from Polymer Source Inc. The polymer was dissolved in hexane (OPTIMA, Fisher) to obtain a PDMS spreading solution at the concentration of $0.44 \mathrm{mg} / \mathrm{ml}$. A PurelabPlus/UV system produced the pure water for the substrate (resistivity $>18.2 \Omega / \mathrm{cm}$ ); and passes the shake test (i.e., small bubbles break as they reach the surface). $13 \mu \mathrm{l}$ of the PDMS solution was deposited on a pure water surface in a Langmuir trough (minitrough, KSV); the hexane evaporates, leaving a partial monolayer behind. A pair of symmetric movable barriers controls the water surface area available to the polymer, and thus the surface concentration. A homemade Brewster angle microscope (BAM) of standard $\operatorname{design}^{27,28}$ images the surface layer. Because the monolayer is unusually thin $(\sim 0.6 \mathrm{~nm}$ compared to $\gtrsim 2 \mathrm{~nm})$, the quality of all optical elements and their mounting are critical. ${ }^{29}$ The monolayer is illuminated with laser light $(532 \mathrm{~nm}$, $200 \mathrm{~mW}$, CrystaLaser) at the Brewster angle, carefully polarized (Glan Tayler polarizer: Lambrecht MGTYE15) in the plane of incidence. Under these circumstances, a perfectly abrupt and smooth Fresnel surface would not reflect light at all. Even a pure water surface reflects, due to its $0.3 \mathrm{~nm}$ rms average roughness; a monolayer will cause additional reflectivity. The reflectivity due to the finite thickness of the PDMS layer is close in magnitude and opposite in phase from the reflectivity due to the roughness of the surface, so that the net reflectivity of the polymer film on (rough) water is almost null, less than that from water alone. ${ }^{12}$ Thus, the polymer monolayer liquid phase reflects less than the polymer gas phase, both on water (see Fig. 1). This is the opposite of the usual contrast, since the reflectivity of a layer is approximately proportional to the square of the amount of material in the layer. The reflected beam was captured by a CCD camera (GPMF602, Panasonic $768 \times 494$ pixels). A PC with an image grabber card finally recorded all the videos at a rate 30 frames/s. The temperature was controlled at $18{ }^{\circ} \mathrm{C}$ with a humidity of $70 \%$. 


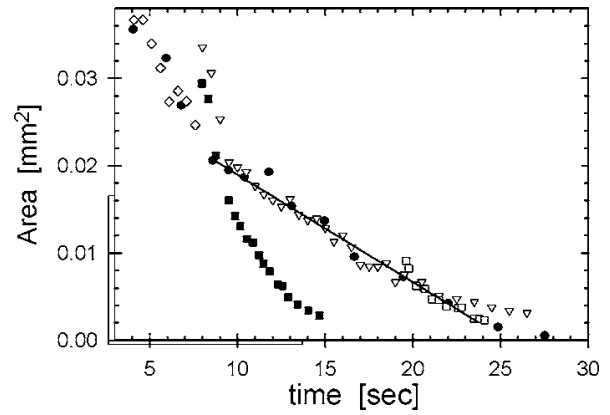

FIG. 6. The area of a hole in a PDMS liquid monolayer as a function of time, for five different domains. A platinum needle was removed from the monolayer, forming the hole. The time for each curve is shifted by $\Delta t$ so that they overlap over the widest range of areas. The straight line is a fit to the two curves which show overlap in area range $0.02-0.002 \mathrm{~mm}^{2}$. The slope of this line is $-1.23 \times 10^{-3} \mathrm{~mm}^{2} / \mathrm{s}$. The initial areas of the hole ranged from $1 \times 10^{-1} \mathrm{~mm}^{2}$ to $1 \times 10^{-2} \mathrm{~mm}^{2}$.

The experiments were performed at a surface concentration of $0.35 \mathrm{mg} / \mathrm{m}^{2}$, where a uniform PDMS monolayer covered $\sim 70 \%$ of the water surface. A platinum wire (diameter $=0.13 \mathrm{~mm}$ ) mounted on a wire holder was well cleaned with $\mathrm{KOH}$ solution and chloroform, then rinsed with pure water. It was carefully manipulated to touch the PDMS monolayer vertically and immediately, smoothly removed. Polymer deposits on the wire; the process may also push polymer away from the wire. The result is that a brighter hole (polymer gas) appears in the dark polymer monolayer liquid (see Fig. 1). This hole always continuously closed until it disappeared. In most cases, the polymer density in the hole did not visibly change; that is, the density remained immeasurably small. In some cases, a bright spot was left behind, probably because of residual contamination trapped in the monolayer where the hole was. The hole area in each frame was determined directly from the number of pixels covered by the whole. Image pixel size $\left(3.63 \times 3.63 \mu \mathrm{m}^{2}\right)$ was determined by imaging a ruled micrometer slide (250 lines/in.) in two orientations, under the same conditions as the experiment.

\section{B. Experimental results}

Preliminary data for the reduction in domain area as a function of time for several domains are given in Fig. 6. The time $t=0$ is shifted from the moment when a hole is formed so that the curves coincide over the widest possible range of areas. We can compare this behavior with the area decrease expected from (38), in dimensional form:

$$
A_{t}=-2 \frac{\lambda}{\eta^{\prime}}\left(1-\frac{1}{\lambda} \sqrt{\frac{A}{\pi}} \Delta \Pi\right) .
$$

In particular, if the difference $\Delta \Pi$ between the internal and external pressures is negligible, then

$$
A_{t}=-2 \lambda / \eta^{\prime}
$$

Indeed, we expect that the area decreases linearly. We observe such a linear decrease for four out of five domains between areas of 0.02 and $0.002 \mathrm{~mm}^{2}$ in which case we determine the line tension as $\lambda=-A_{t} \eta^{\prime} / 2=0.65 \pm 0.03 \mathrm{pN}$, using $\eta^{\prime}=1.063 \times 10^{-3} \mathrm{~kg} / \mathrm{m} \mathrm{s}^{30}$ Note that if one looks at in- dividual area curves, one can find values of $A_{t}$ up to a factor 3 larger or smaller at shorter or longer times, respectively, but, unlike $A_{t}$ in the central portion, these values are different for different domains. The initial more rapid decrease in area, as well as the very different behavior of one domain, probably represent transients due to the hole-formation process. Also note that the domain shapes are not strictly circular, as assumed in this analysis; domain edges may be rough, or the domain may be tear-shaped, for example, and not relax to the equilibrium circular shape in the time it takes for the domains to close. Furthermore, one of the domains was very near another, larger domain. These data must thus be taken as preliminary.

The line tension tentatively determined in this manner should be compared to the line tension measured from the relaxation of domains towards a circle, $1.1 \pm 0.3 \mathrm{pN} .{ }^{16}$ Note that the error estimate is from the experimental spread; an additional uncertainty arises from the model used to relate relaxation times to line tensions. The only such model available $^{19}$ applies to liquid-liquid coexistence, and in particular assumes that both fluids act incompressibly. The hole closing results here suggest that this model is invalid for PDMS gas-liquid coexistence. We are presently treating relaxation of fluid domains assuming that the gas domain is infinitely compressible. Considering the limits of both experiment and analysis, the agreement between the two values, within a factor of 2 , must be considered very suggestive.

One can further speculate about the slowing down of the hole closing at smaller areas seen in some cases. As the hole closes, one should consider the fate of any polymer or other material at the center of the hole. Any polymer within the hole would tend to accrete on the hole edge; the material involved should be too small to be noticeable. In one of the domains, a three-dimensional speck of material, which must therefore represent contamination, was left as the domain closed entirely. If the amount of material in the hole remains fixed, as one would expect if the material is foreign to the monolayer, its density will increase to the point that a significant pressure must appear in the domain. A first estimate of this effect can be given assuming that the material follows the ideal gas law with $\Delta \Pi A=N k T$, with the number of molecules $N$ in the hole held fixed. This leads to the equation

$$
A_{t}=-2 \frac{\lambda}{\eta^{\prime}}\left(1-\frac{N k T}{\lambda \sqrt{\pi A}}\right) .
$$

In this case, the collapse of the hole slows and in fact ceases when $A=(N k T)^{2} / \lambda^{2} \pi$. If $N$ can be controlled, this offers an additional way to estimate $\lambda$.

\section{CONCLUSIONS}

The explicit functions (38) and (46) derived here for the area evolution of a circular hole in an incompressible twodimensional liquid coupled to a fluid substrate can thus be compared directly to the area evolution of a polymer Langmuir monolayer. The line tension deduced from preliminary data is within a factor of 2 of independent experimental de- 
terminations of that line tension, which, given the uncertainties of both determinations, must be considered very reasonable agreement.

The closing of such holes must involve a vertical component of the fluid velocity, due to the continuity and incompressibility of the substrate. Most models of Langmuir monolayer hydrodynamics assume strictly horizontal flow. Here, from explicit expression (43) for the stream function, and thus the flow, we see that while surface tension forces the flow to be horizontal at the surface, the vertical component of the velocity is of the same order of magnitude as the horizontal one within the substrate. In fact, the flow has a stagnation point at the center of the domain, and is nearly vertical in the area directly below the hole and at depths of the order of the hole radius. It would be interesting to visualize such flow experimentally.

One limitation of the present model is that it assumes a circular hole, $\partial \Omega_{i}$. It is quite difficult to form a strictly circular hole experimentally, and in these experiments, the holes did not have time to relax to a circular shape before they disappeared. Another limitation of the model is that it ignores accretion on and evaporation from the edge of the liquid domain. In the absence of such accretion, the polymer density within the hole will change with time. ${ }^{20}$ In our experiments, the polymer density in the hole did not visibly change; that is, it remained immeasurably small. However, at some point, either accretion on the edge or nucleation within the hole must occur. If either evaporation or accretion played important roles, the rate of hole closing would depend on the initial size of the hole. In fact over a significant range of domain area, the same rate was observed for domains whose initial size varied by a factor of 10 , and no nucleation was observed. The model does clearly capture the basic holeclosing behavior for this polymer monolayer. It will be interesting to consider the effect of $\Delta \Pi \neq 0$, which may also be related to the slowing down of the hole closing at smaller areas seen in some cases. Also, holes within monolayers of smaller molecules may behave quite differently. It would also be interesting to study the effect of long range forces, which would become relevant with larger holes or a smaller line tension. Note that the importance of long range forces is exponentially sensitive to the line tension [Eq. (1)].

The mass-balance formula, which takes into account accretion and evaporation, is that for the boundary of the hole, $\partial \Omega_{i}$, and includes mass transport along $\partial \Omega_{i}$ and the flux along the normal to the boundary from $\Omega$ and $\Omega^{h}$. This is a complication that will be added to the theory if accretion or evaporation is observed experimentally.

The experimental results strongly suggests that the twodimensional polymer gas cannot support a stress at these time scales. We conclude that the Gibbs elasticity is $K_{g} \ll 10^{-5} \mathrm{mN} / \mathrm{m}$. This is about an order of magnitude lower than the limit that could be established by other experimental means. These results demonstrate that for Langmuir layers with gas phases it is necessary to both account for the compressibility of the gas phase and the associated vertical motion induced in the subfluid. We are in the process of developing a theory for domain relaxation valid under the current assumptions.

\section{ACKNOWLEDGMENT}

E.K.M. and L.Z. were partially supported by the National Science Foundation under Grant No. 9984304.

${ }^{1}$ A. W. Adamson and A. P. Gast, Physical Chemistry of Surfaces, 6th ed. (Wiley, New York, 1998).

${ }^{2}$ K. Simons and E. Ikonen, "Functional rafts in cell membranes," Nature (London) 387, 569 (1997).

${ }^{3}$ M. Edidin, "The state of lipid rafts: From model membranes to cells," Annu. Rev. Biophys. Biomol. Struct. 32, 257 (2003).

${ }^{4}$ S. Mayor and M. Rao, "Rafts: Scale-dependent, active lipid organization at the cell surface," Traffic Eng. 5, 231 (2004).

${ }^{5}$ R. G. Parton and J. F. Hancock, "Lipid rafts and plasma membrane microorganization: Insights from Ras," Trends Cell Biol. 14, 141 (2004).

${ }^{6}$ S. Mukherjee and F. R. Maxfield, "Membrane domains," Annu. Rev. Cell Dev. Biol. 20, 839 (2004).

${ }^{7}$ R. De Koker and H. M. McConnell, "Hydrodynamics of domain size equilibration in monolayers," J. Phys. Chem. B 102, 6927 (1998).

${ }^{8}$ Z. Khattari, E. Hatta, P. Heinig, P. Steffen, Th. M. Fischer, and R. Bruinsma, "Cavitation of Langmuir monolayers," Phys. Rev. E 65, 041603 (2002).

${ }^{9}$ E. K. Mann and D. Langevin, "Poly(dimethylsiloxane) molecular layers at the surface of water and of aqueous surfactant solutions," Langmuir 7, 1112 (1995).

${ }^{10}$ E. K. Mann, "PDMS films at water surfaces: texture and dynamics," Ph.D. thesis, Université de Paris VI (1992).

${ }^{11}$ L. T. Lee, E. K. Mann, D. L. Langevin, and B. Farnoux, "Neutron reflectivity and ellipsometry studies of a polymer monolayer spread on the water surface," Langmuir 7, 3076 (1991).

${ }^{12}$ E. K. Mann, S. Hénon, D. Langevin, and J. Meunier, "Molecular layers of a polymer at the free water surface: Microscopy at the Brewster angle," J. Phys. II 2, 1683 (1992).

${ }^{13}$ P. J. Winch and J. C. Earnshaw, "A light scattering study of phase transitions in monolayers of $n$-pentadecanoic acid," J. Phys.: Condens. Matter 1, 7187 (1989).

${ }^{14}$ R. De Koker and H. M. McConnell, "Circle to dogbone-shapes and shape transitions of lipid monolayer domains," J. Phys. Chem. 97, 13419 (1993).

${ }^{15}$ E. K. Mann and S. V. Primak, "Stability of two-dimensional foams in Langmuir monolayers,” Phys. Rev. Lett. 83, 5397 (1999).

${ }^{16}$ E. K. Mann, S. Hénon, D. Langevin, J. Meunier, and L. Léger, "The hydrodynamics of domain relaxation in a polymer monolayer," Phys. Rev. E 51, 5708 (1995).

${ }^{17}$ E. K. Mann and S. Primak, "The stability of two-dimensional foams in Langmuir monolayers,” Phys. Rev. Lett. 83, 5397 (1999).

${ }^{18}$ D. K. Lubensky and R. E. Goldstein, "Hydrodynamics of monolayer domains at the air-water interface," Phys. Fluids 8, 843 (1996).

${ }^{19}$ H. A. Stone and H. M. McConnell, "Hydrodynamics of quantized shape transitions of lipid domains," Proc. R. Soc. London, Ser. A 448, 97 (1995).

${ }^{20}$ See EPAPS Document No. E-PHFLE6-18-017606 for appendices. This document can be retrieved via a direct link in the online article's HTML reference section or via the EPAPS homepage (http://www.aip.org/ pubservs/epaps.html).

${ }^{21}$ D. J. Benvegnu and H. M. McConnell, "Line tension between liquid domains in lipid monolayers," J. Phys. Chem. 96, 6820 (1992).

${ }^{22}$ J. Lauger, C. R. Robertson, C. W. Frank, and G. G. Fuller, "Deformation and relaxation processes of mono- and bilayer domains of liquid crystalline Langmuir films on water," Langmuir 12, 5630 (1996).

${ }^{23}$ S. Wurlitzer, P. Steffen, and Th. M. Fischer, "Line tension in Langmuir monolayers probed by point forces," J. Chem. Phys. 112, 5915 (2000).

${ }^{24}$ S. Wurlitzer, P. Steffen, M. Wurlitzer, Z. Khattari, and Th. M. Fischer, "Line tension in Langmuir monolayers probed by point forces," J. Chem. Phys. 113, 3822 (2000). 
${ }^{25}$ S. Granick, "Surface pressure of linear and cyclic poly(dimethylsiloxane) in the transition region," Macromolecules 18, 1597 (1985).

${ }^{26}$ I. S. Gradshteyn and I. M. Ryzhik, Table of Integrals, Series, and Products, 6th ed. (Academic, San Diego, 2000).

${ }^{27}$ S. Hénon and J. Meunier, "Microscopy at the Brewster angle: Direct observation of first-order phase transitions in monolayers," Rev. Sci. Instrum. 62, 936 (1991).

${ }^{28}$ D. Hönig and D. Möbius, "Direct visualization of monolayers at the air- water interface by Brewster angle microscopy," J. Phys. Chem. 95, 4590 (1991).

${ }^{29}$ L. Zou, J. Wang, V. Beleva, E. Kooijman, S. Primak, J. Risse, W. Weissflog, A. Jakli, and E. K. Mann, "Langmuir monolayers of bent-core molecules," Langmuir 20, 2772 (2004).

${ }^{30}$ CRC Handbook of Chemistry and Physics, 83 rd ed., edited by D. R. Ride (CRC Press, Boca Raton, 2002). 